\title{
Targeted next-generation sequencing in Slovak cardiomyopathy patients
}

\author{
Nagyova $E^{1,2,6}$, Radvanszky J ${ }^{4,5}$, Hyblova $M^{1,6}$, Simovicova $V^{7}$, Goncalvesova $E^{7}$, \\ Asselbergs $\mathrm{FW}^{2,3,8}$, Kadasi $\mathrm{L}^{1,5}$, Szemes $\mathrm{T}^{1,4,6}$, Minarik $\mathrm{G}^{1,4,6}$ \\ Department of Molecular Biology, Faculty of Natural Sciences, Comenius University in Bratislava, Bratislava, Slovakia. \\ E.Nagyova@umcutrecht.nl
}

\begin{abstract}
OBJECTIVES: For the first time we used targeted next-generation sequencing to detect candidate pathogenic variants in Slovak cardiomyopathy patients.

BACKGROUND: Targeted next-generation sequencing is considered to be the best practice in genetic diagnostics of cardiomyopathies. However, in Slovakia, with high cardiomyopathies prevalence of $1 / 440$, the current diagnostic tests are still based on Sanger sequencing of a few genes. Consequently, little is known about the exact contribution of pathogenic variants in known cardiomyopathy genes in Slovak patients.

METHODS: We used a panel of 46 known cardiomyopathy-associated genes to detect genetic variants in 16 Slovak cardiomyopathy patients (6 dilated, 8 hypertrophic, 2 non-compaction subtypes).

RESULTS: We identified candidate pathogenic variants in 11 of 16 patients (69\%). Genes with higher count of candidate pathogenic variants were MYBPC3, MYH and TTN, each with 3 different variants. Seven variants ACTC1 (c.329C>T), ANKRD1 (c.683G>T), MYH7 (c.1025C>T), PKP2 (c.2003delA), TTN (c.51655C>T, c.84841G>T, c.101874_101881delAGAATTTG) have been detected for the first time and might represent Slovak-specific genetic cause.

CONCLUSIONS: We have performed genetic testing of previously untested Slovak cardiomyopathy patients using next-generation sequencing cardiomyopathy gene panel. Given the high percentage of candidate pathogenic variants it should be recommended to implement this method into routine genetic diagnostic practice in Slovakia (Tab. 4, Ref. 39). Text in PDF www.elis.sk.

KEY WORDS: cardiomyopathy, Slovak patients, next-generation sequencing, gene panel, genetic testing.
\end{abstract}

Abbreviations: ENA - European Nucleotide Archive, CMs -
Cardiomyopathies, NGS - Next-generation sequencing, DCM -

${ }^{1}$ Department of Molecular Biology, Faculty of Natural Sciences, Comenius University in Bratislava, Bratislava, Slovakia, ${ }^{2}$ Department of Cardiology, Division Heart \& Lungs, UMC Utrecht, University of Utrecht, Utrecht, Netherlands, ${ }^{3}$ Durrer Center for Cardiovascular Research, Netherlands Heart Institute, Amsterdam, Netherlands, ${ }^{4} \mathrm{Geneton}$ Ltd., Bratislava, Bratislava, Slovakia, ${ }^{5}$ Institute for Clinical and Translational Research, Biomedical Research Centre, Slovak Academy of Sciences, Bratislava, Slovakia, ${ }^{6}$ Medirex Inc., Bratislava, Slovakia, ${ }^{7}$ Heart Failure and Transplant Department, National Cardiovascular Institute, Bratislava, Slovakia, and ${ }^{8}$ Institute of Cardiovascular Science, Faculty of Population Health Sciences, University College London, London, United Kingdom

Address for correspondence: E. Nagyova, MSc, Department of Molecular Biology, Faculty of Natural Sciences, Comenius University in Bratislava, Ilkovicova 6, SK-842 15 Bratislava 4, Slovakia.

Phone: +31.8875 .57381$

Acknowledgements: E. Nagyova was supported by The National Scholarship Programme of Slovak Republic for the Support of Mobility of Students, PhD Students, University Teachers and Researchers. Folkert W. Asselbergs is supported by a Dekker scholarship-Junior Staff Member 2014T001 - Netherlands Heart Foundation and UCL Hospitals NIHR Biomedical Research Centre. This work was supported as part of the project REVOGENE - Research center for molecular genetics (ITMS 26240220067) supported by the Research \& Development Operational Programme funded by the ERDF.
Dilated cardiomyopathy, HCM - Hypertrophic cardiomyopathy, NNCM - Non-compaction cardiomyopathy, MSR - MiSeq Reporter, BWA - Burrows-Wheeler Aligner, GATK - Genome Analysis Toolkit, ExAC - The Exome Aggregation Consortium, AoCGV - Atlas of Cardiac Genetic Variation, ACMG - American College of Medical Genetics and Genomics, AMP - Association for Molecular Pathology.

\section{Introduction}

Cardiomyopathies (CMs) are a heterogeneous group of disorders of cardiac muscle with high morbidity and mortality, leading to chronic end-stage heart failure or sudden cardiac death (1). In cardiomyopathies, mutations have been detected in more than 40 genes that encode the crucial elements of cardiomyocytes, such as sarcomeric filaments, calcium-metabolizing proteins, desmosomes, and mitochondrial enzymes $(2,3)$.

Mutation detection based on next-generation sequencing (NGS) of gene panels of known CM genes is currently considered the best practice for genetic analysis in CMs (4). Knowledge about genetic cause of patient's CM can help to predict disease prognosis, to indicate treatment, such as the use of cardiac devices, or to recognize family members at risk even before the disease has clinically manifested $(5,6)$. 
Tab. 1. Clinical characterization of the Slovak patient cohort.

\begin{tabular}{|c|c|c|c|c|c|c|c|}
\hline Sample & $\begin{array}{c}\text { Candidate } \\
\text { pathogenic } \\
\text { variants }(\mathrm{No})\end{array}$ & Sex & CM subtype & $\begin{array}{l}\text { Age presentation/ } \\
\text { last evaluation }\end{array}$ & $\begin{array}{c}\text { Transplantation (age)/ } \\
\text { Death (age) }\end{array}$ & $\begin{array}{l}\text { LV ejection } \\
\text { fraction }(\%)\end{array}$ & $\begin{array}{l}\text { Affected family } \\
\text { members }\end{array}$ \\
\hline 1 & 1 & $\mathrm{M}$ & $\mathrm{DCM}$ & $46 / 53$ & $\mathrm{Y}(50) / \mathrm{N}$ & $21^{1}$ & N/A \\
\hline 2 & 1 & $\mathrm{M}$ & DCM & $26 / 38$ & WL/Y(N/A) & 15 & N/A \\
\hline 3 & 2 & $\mathrm{M}$ & DCM & $40 / 46$ & $\mathrm{~N} / \mathrm{N}$ & 25 & $\mathrm{~N}$ \\
\hline 4 & 1 & $\mathrm{M}$ & DCM & $52 / 59$ & $\mathrm{Y}(58) / \mathrm{N}$ & $20^{1}$ & $\mathrm{Y}$ \\
\hline 5 & 0 & $\mathrm{M}$ & DCM & $46 / 52$ & $\mathrm{~N} / \mathrm{N}$ & 20 & $\mathrm{Y}$ \\
\hline 6 & 1 & $\mathrm{~F}$ & DCM & $48 / 48$ & $\mathrm{~N} / \mathrm{N}$ & 45 & $\mathrm{~N}$ \\
\hline 7 & 0 & $\mathrm{~F}$ & $\mathrm{HCM}$ & $74 / 74$ & $\mathrm{~N} / \mathrm{N}$ & 64 & $\mathrm{Y}$ \\
\hline 8 & 2 & $\mathrm{~F}$ & $\mathrm{HCM}$ & $12 / 38$ & $\mathrm{~N} / \mathrm{N}$ & normal & $\mathrm{Y}$ \\
\hline 9 & 0 & $\mathrm{M}$ & $\mathrm{HCM}$ & $27 / 55$ & $\mathrm{~N} / \mathrm{N}$ & 35 & $\mathrm{~N} / \mathrm{A}$ \\
\hline 10 & 1 & $\mathrm{M}$ & $\mathrm{HCM}$ & $25 / 37$ & $\mathrm{WL} / \mathrm{N}$ & 65 & $\mathrm{Y}$ \\
\hline 11 & 2 & $\mathrm{M}$ & $\mathrm{HCM}$ & $59 / 59$ & $\mathrm{~N} / \mathrm{N}$ & 50 & N/A \\
\hline 12 & 0 & $\mathrm{~F}$ & $\mathrm{HCM}$ & $62 / 66$ & $\mathrm{~N} / \mathrm{N}$ & 70 & $\mathrm{~N} / \mathrm{A}$ \\
\hline 13 & 1 & $\mathrm{M}$ & $\mathrm{HCM}$ & $38 / 42$ & $\mathrm{~N} / \mathrm{N}$ & 60 & $\mathrm{Y}$ \\
\hline 14 & 2 & $\mathrm{M}$ & $\mathrm{HCM}$ & $23 / 23$ & $\mathrm{~N} / \mathrm{N}$ & 65 & $\mathrm{Y}$ \\
\hline 15 & 1 & $\mathrm{M}$ & NNCM & $20 / 23$ & $\mathrm{~N} / \mathrm{N}$ & 45 & N/A \\
\hline 16 & 0 & $\mathrm{M}$ & NNCM & $42 / 42$ & $\mathrm{~N} / \mathrm{N}$ & 17 & N/A \\
\hline
\end{tabular}

$\mathrm{M}$ - male; F - female; CM - cardiomyopathy; NNCM - non-compaction cardiomyopathy ; DCM - dilated cardiomyopathy; HCM - hypertrophic cardiomyopathy; LV - left ventricle; N/A - not available; $\mathrm{N}$ - no; $\mathrm{Y}$ - yes; WL - waiting list; ${ }^{1}$ - before transplantation

In Slovakia, data on genetic etiology of cardiomyopathies in general is largely missing. In the 2013 report of the Slovak national center of health information, there were 12,317 patients with known cardiomyopathy (7,680 men and 4,637 women) which represents prevalence of 1/440 (National Health Information Center (7). During years 2010 and 1998, 1000 patients with heart failure referred by cardiologists to a single tertiary center in order to assess the indication for heart transplantation were hospitalized and monitored ( $86.8 \%$ men and $13.2 \%$ women, mean age 49.0 \pm 10.9 years). The majority of patients $(80.8 \%)$ had severe left ventricular systolic dysfunction with ejection fraction $<30 \% ; 1.8$ $\%$ of patients had preserved left ventricular ejection fraction $\geq 50$ $\%$ (8). Despite of the number of CM patients and the availability of NGS methods, the current diagnostic tests in Slovakia are still based on Sanger sequencing of a few genes (9).

Here, we have performed genetic testing of previously untested Slovak cardiomyopathy patients using standard NGS cardiomyopathy gene panel in order to demonstrate the beneficial effect of implementing modern methods in diagnostic practice.

\section{Material and methods}

\section{Patient information}

All patients signed an informed consent to participate in the study. The genomic DNA samples from 6 dilated (DCM), 8 hypertrophic (HCM) and 2 non-compaction (NNCM) cardiomyopathy patients (Tab. 1) were obtained from the Heart Failure and Transplant Department, National Cardiovascular Institute, Bratislava, Slovakia.

\section{Next-generation sequencing}

Genomic DNA was extracted from whole blood according to standard procedures and samples were purified by ZYMO
DNA Clean \& Concentrator ${ }^{\mathrm{TM}}-5$ preps (Zymo Reasearch, USA) according to instructions of the manufacturer. Further, 50ng of DNA from each sample was processed into adapter-tagged DNA library according to the TruSight Rapid Capture (Illumina, San Diego, CA, USA) guide including tagmentation, purification, first amplification, pre-enrichment pooling, 2 steps of enrichment and final amplification of the library. In enrichment steps, coding exons of 46 cardiomyopathy genes (ABCC9, ACTC1, ACTN2, ANKRD1, CASQ2, CAV3, CRYAB, CSRP3, CTF1, DES, DSC2, DSG2, DSP, DTNA, EMD, FHL2, GLA, JUP, LAMA4, LAMP2,

Tab. 2. Amount of variants after filtering.

\begin{tabular}{cccccc}
\hline Sample & VCF all & $\begin{array}{c}\text { VCF } \\
\text { PASS }\end{array}$ & $\begin{array}{c}\text { VCF PASS }+ \\
\text { VCF GQ 99 }\end{array}$ & $\begin{array}{c}\text { After } \\
\text { filters }\end{array}$ & $\begin{array}{c}\text { Rare } \\
\text { variants }\end{array}$ \\
\hline 1 & 164 & 146 & 146 & 1 & 1 \\
\hline 2 & 131 & 115 & 115 & 2 & 1 \\
\hline 3 & 123 & 98 & 98 & 4 & 2 \\
\hline 4 & 181 & 147 & 128 & 2 & 2 \\
\hline 5 & 185 & 118 & 103 & 1 & 0 \\
\hline 6 & 187 & 132 & 113 & 2 & 1 \\
\hline 7 & 173 & 83 & 66 & 0 & 0 \\
\hline 8 & 123 & 100 & 87 & 3 & 2 \\
\hline 9 & 156 & 139 & 139 & 1 & 0 \\
\hline 10 & 161 & 146 & 145 & 1 & 1 \\
\hline 11 & 143 & 132 & 132 & 2 & 2 \\
\hline 12 & 229 & 96 & 75 & 1 & 1 \\
\hline 13 & 130 & 95 & 95 & 1 & 1 \\
\hline 14 & 153 & 124 & 118 & 2 & 2 \\
\hline 15 & 152 & 109 & 106 & 3 & 1 \\
\hline 16 & 151 & 104 & 101 & 0 & 0 \\
\hline Mean & 158.9 & 117.8 & 110.4 & 1.6 & 1.1 \\
\hline STDEV & 27.9 & 20.9 & 23.8 & 1.1 & 0.8 \\
\hline VESS & -15 & 13 &
\end{tabular}

VCF PASS - passed all quality filters; GQ - Genotype Quality; Rare variants - information from Atlas of Cardiac Genetic Variation 
46-51

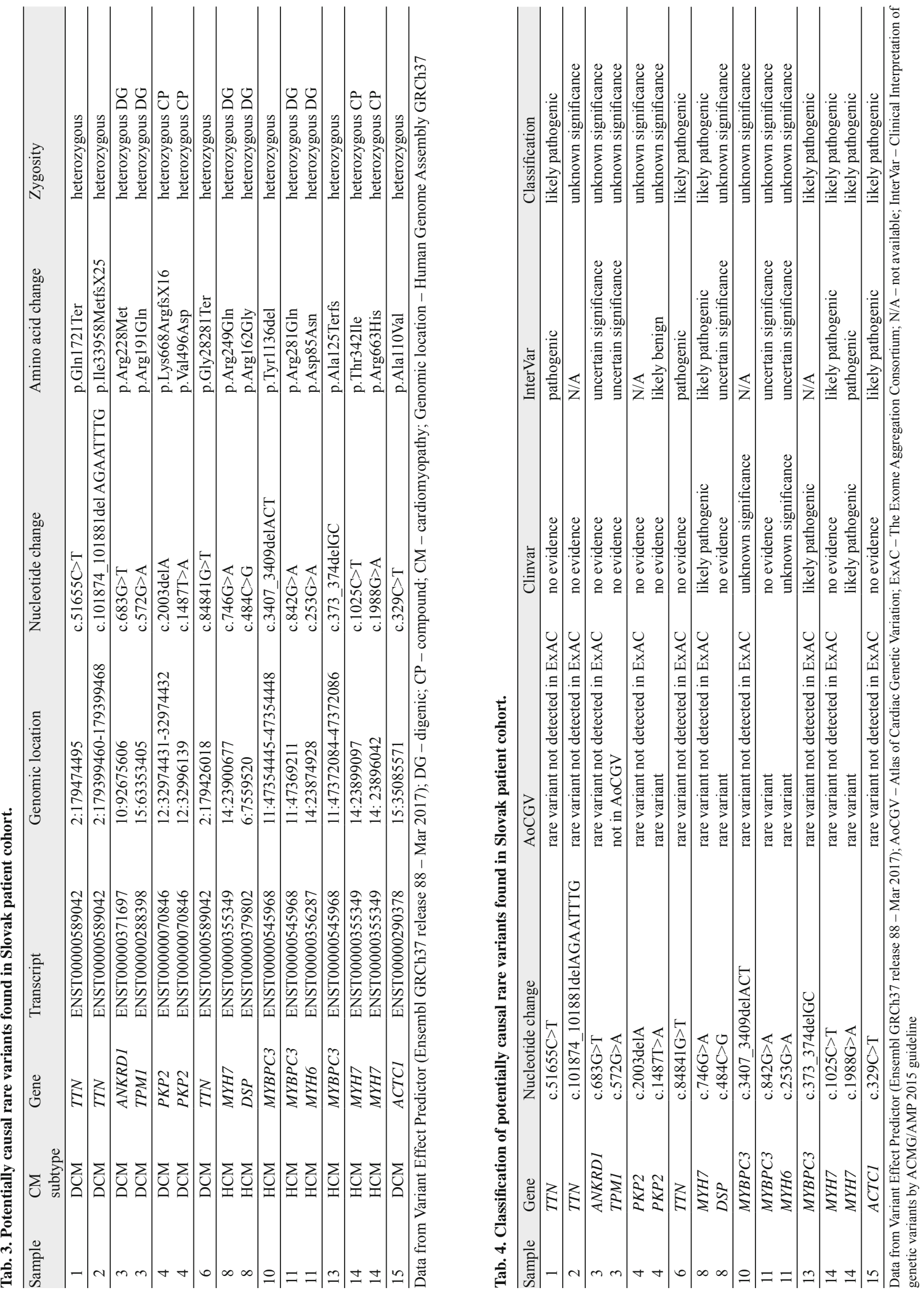


LDB3, LMNA, MYBPC3, MYH6, MYH7, MYL2, MYL3, MYLK2, MYOZ2, NEXN, PKP2, PLN, PRKAG2, RBM20, RYR2, SGCD, TAZ, TCAP, TMEM43, TNNC1, TNNI3, TNNT2, TPM1, TTN, TTR, $V C L$ ) were enriched using the TruSight Cardiomyopathy Sequencing Panel oligos (Illumina, San Diego, CA, USA).

Concentration of libraries was measured using Qubit ${ }^{\circledR}$ dsDNA HS kit (Invitrogen, CA, USA). Template size distribution was verified using Agilent ${ }^{\mathbb{R}}$ HighSensitivity DNA Chip and 2100 Bioanalyser (Agilent Technologies, Germany). Finally, DNA templates were normalized to $4 \mathrm{nM}$ concentration, denaturated by $0.2 \mathrm{~N} \mathrm{NaOH}$ (SIGMA-ALDRICH, Germany). Bridge PCR amplification and sequencing run of the panel of genes was then performed on the pooled samples of our 16 patients in two separate runs on MiSeq (Illumina, San Diego, CA, USA) using paired-end setting of 2x150bp reads.

\section{Data analysis and interpretation}

Sequence data generated from TruSight Cardiomyopathy enriched libraries were analyzed by the on-instrument MiSeq Reporter (MSR) software. The samples were demultiplexed and fastq files were generated. The software used the Burrows-Wheeler Aligner (BWA) (10) to align the reads against the human reference genome GRCh37/hg19 to create bam files, and the Genome Analysis Toolkit (GATK) (11) to perform variant analysis of the target regions specified in the manifest file to produce vef files. Filtering criteria used in vef files were coverage of the genetic position at least 20x, minimum genotype quality $>99$, variant frequency (percentage of reads supporting the alternate allele) $>0.20$, locus genotype quality $<10.0000$ or not present, indel repeat length $>$ 8 , strand bias $>-10$, site genotype conflicts with proximal indel call removed, site mapping quality $<0.0000$, locus quality score normalized by allele depth $<0.0000$.

Ensembl Variant Effect Predictor software (12) was used to annotate variants in filtered vcf files. We filtered out annotated variants with population frequency $>0.5 \%$ according to the data from ExAC database (The Exome Aggregation Consortium) (13), using reference data from non-Finland European population. We also filtered out data with other than missense, frameshift, splice region, in-frame deletions, stop gain and stop loss consequence. Because of the controversial interpretation (14) missense variants in the TTN gene were listed separately. For the selection of candidate causative mutations we have considered data from Atlas of Cardiac Genetic Variation (AoCGV) (15) and from ClinVar databases (16) while prioritized variants referred as rare and/or pathogenic. The clinical interpretation of genetic variants by American College of Medical Genetics and Genomics and the Association for Molecular Pathology (ACMG/AMP) 2015 guidelines was made using online tool InterVar (17). We classified variants referred in ClinVar database or after InverVar classification as "pathogenic" or "likely pathogenic" as likely pathogenic and other variants as variants of unknown significance.

\section{Results}

We analyzed genomic DNA samples from 16 cardiomyopathy patients (6 dilated - DCM, 8 hypertrophic - HCM and 2 non- compaction - NNCM) (Tab. 1) using NGS workflow including the TruSight Cardiomyopathy panel of 46 selected genes.

The mean region coverage depth of the sequenced libraries was comparable between the two produced runs: $283 \mathrm{x} \pm 108$ and $308 \mathrm{x} \pm 301$. Target coverage at the sequencing depth of $20 \mathrm{x}$ was $99.6 \% \pm 0.4$ for the first run and $95.8 \% \pm 4.4$ for the second.

On average, $159 \pm 28$ variants were detected per patient. After filtering we obtained $1.6 \pm 1$ variants per patient (Tab. 2). In total, 26 variants were considered for further assessment.

We identified potentially causal variants in 11 of 16 patients (69\%) in nine known genes (Tab. 3). All selected variants were classified in AoCGV as 'rare variants' or they were not previously detected in the ExAC, the 1000 Genomes or the Exome Sequencing Project database (> 70000 samples).

According to ACMG/AMP 2015 guidelines and data from ClinVar database we classified seven variants as likely pathogenic and nine variants as variants of unknown significance (Tab. 4). The most frequently mutated genes were $M Y B P C 3, M Y H$, and TTN (three different variants).

Six of eleven patients were carrying one heterozygous variant, 3 patients were carrying two heterozygous variants in two different genes and 2 patients have shown compound heterozygosity (Table 3).

Four 'rare variants' ( $D S P$ c. $484 \mathrm{C}>\mathrm{G}$; MYBPC3 c.842G $>\mathrm{A}$; PKP2 c. $1487 \mathrm{~T}>\mathrm{A}$; TPM1 c.572G $>\mathrm{A}$ ) and seven 'rare variants, previously not detected in the ExAC database' (ACTC1 c.329C>T; ANKRD1 c.683G > T; MYH7 c. $1025 \mathrm{C}>\mathrm{T}$; PKP2 c. $1459 \mathrm{C}>\mathrm{T}$, c. $2003 \mathrm{delA}$; TTN c. $51655 \mathrm{C}>\mathrm{T}$, c. $84841 \mathrm{G}>\mathrm{T})$ that were detected in our cohort, have, to our knowledge, not been reported previously neither as a disease-causing or as a benign polymorphism (Tab. 4).

\section{Discussion}

Due to the large variation in clinical manifestations of HCM and DCM, ranging from asymptomatic forms to progressive heart failure and sudden cardiac death, not all individuals can be diagnosed properly relying solely on phenotype characteristics. Genetic testing provides clues to predict disease prognosis, to indicate treatment, such as the use of cardiac devices, and to recognize family members at risk. Here, we used a standard NGS panel of 46 cardiomyopathy genes to determine the genetic yield in 16 Slovak patients for the first time.

Seven rare variants that have been detected in our cohort were previously not detected in ExAC database (ACTC1 c.329C $>\mathrm{T}$; ANKRD1 c. $683 \mathrm{G}>\mathrm{T} ;$ MYH7 c. $1025 \mathrm{C}>\mathrm{T} ;$ PKP2 c. $1459 \mathrm{C}>\mathrm{T}$, c. 2003 delA; TTN c. $51655 \mathrm{C}>\mathrm{T}$, c. $84841 \mathrm{G}>\mathrm{T}$ ) and to our knowledge they have not been reported previously as a disease cause or as a benign polymorphism. However, without population specific genotype databases such as the Dutch GoNL database (18), it is difficult to evaluate if these might represent population-specific variants in Slovakia.

Three variants (MYH7 c.746G >A; MYBPC3 c.373_374delGC, c.3407_3409delACT) were previously not detected in the ExAC database but they are mentioned in the ClinVar database. 
46-51

We classified 7 of 16 candidate pathogenic variants as likely pathogenic (ACTC1 c.329C>T; MYBPC3 c.373_374delGC; MYH7 c. $746 \mathrm{G}>$ A, c. $1988 \mathrm{G}>\mathrm{A}$, c. $1025 \mathrm{C}>\mathrm{T} ;$ TTN c. $51655 \mathrm{C}>\mathrm{T}$, c. $84841 \mathrm{G}>\mathrm{T})$ and nine variants as variants of unknown significance (ANKRD1 c.683G > T; DSP c.484C >G; MYBPC3 c.842G >A; MYH6 c.253G $>$ A; $P K P 2$ c.2003delA, c. $1487 \mathrm{~T}>$ A; TPM1 c.572G $>$ A; TTN c.101874_101881delAGAATTTG, c.84841G $>$ T).

In other studies the diagnostic yield of NGS cardiomyopathy panels was at $35.2 \%$ in Finish cohort (145 permanent pacemaker patients) (37), 33.3\% in Russian cohort (38 cardiomyopathy patients) (38), $45 \%$ in Tunisian cohort (11 HCM patients) (39). The higher diagnostic yield in our cohort $(69 \%)$ could be influenced by the fact that we have selected patients with the most severe cardiomyopathy phenotype which were not previously genetically tested. In countries where genetic testing in cardiomyopathies is a routine practice the most common genetic causes are already tested in many patients and they are not necessary included in new studies. Furthermore, variants selected in our study are candidate pathogenic variants and we expect the real diagnostic yield to be lower after further analysis, e.g. segregation analysis in patient families, which was not possible in our study.

In Slovakia there is an urgent need to modernize genetic diagnostic practice. Even genes already well known to be associated with cardiomyopathies are not routinely tested in Slovak patients. Slovak population might be largely underdiagnosed and borderline cardiomyopathy patients might be classified incorrectly. Given the high percentage of candidate pathogenic variants in other countries as well as in our preliminary data $(69 \%)$ it should be recommended to implement NGS cardiomyopathy gene panels in routine genetic diagnostic practice also in Slovakia. The detection of pathogenic variants is especially important for cascade screening in families and identification of family members in risk which should be regularly checked by cardiologist to get proper treatment on time or even to prevent sudden cardiac death. One of the most important steps to make progress in cardiology routine testing is to increase the knowledge of cardiologist about benefits connected with proper genetic testing for patients and their families and make those tests widely available by involving the insurance companies, as it is common in other countries.

\section{References}

1. Wilde AA, Behr ER. Genetic testing for inherited cardiac disease. Nat Rev Cardiol 2013; 10 (10): 571-583.

2. Hershberger RE, Hedges DJ, Morales A. Dilated cardiomyopathy: the complexity of a diverse genetic architecture. Nat Rev Cardiol 2013; 10 (9): 531-547.

3. Maron BJ, Maron MS, Semsarian C. Genetics of hypertrophic cardiomyopathy after 20 years: clinical perspectives. J Am Coll Cardiol 2012; 60 (8): 705-715.

4. Mogensen J, van Tintelen JP, Fokstuen S et al. The current role of next-generation DNA sequencing in routine care of patients with hereditary cardiovascular conditions: a viewpoint paper of the European Society of Cardiology working group on myocardial and pericardial diseases and members of the European Society of Human Genetics. Eur Heart J 2015; 36 (22): 1367-1370.
5. Meune C, Van Berlo JH, Anselme F, Bonne G, Pinto YM, Duboc D. Primary prevention of sudden death in patients with lamin $\mathrm{A} / \mathrm{C}$ gene mutations. N Engl J Med 2006; 354 (2): 209-210.

6. Jansweijer JA, Nieuwhof K, Russo F et al. Truncating titin mutations are associated with a mild and treatable form of dilated cardiomyopathy. Eur J Heart Fail 2017; 19 (4): 512-521.

7. National Health Information Center, Activity of cardiology clinics in Slovakia in 2013. Edition Health Statistics 2014.

8. Lesny P, Luknar M, Matejka M et al. Ten-year survival and prognostic markers in one thousand patients with advanced heart failure. A singlecentre analysis. Biomed Pap Med Fac Univ Palacky Olomouc Czech Repub 2016; 160 (2): 257-262.

9. Priganc M, Zigova M, Boronova I et al. Analysis of SCN5A Gene Variants in East Slovak Patients with Cardiomyopathy. J Clin Lab Anal 2017; $31(2)$.

10. Li H, Durbin R. Fast and accurate long-read alignment with BurrowsWheeler transform. Bioinformatics 2010; 26 (5): 589-595.

11. McKenna A, Hanna M, Banks E et al. The Genome Analysis Toolkit: a MapReduce framework for analyzing next-generation DNA sequencing data. Genome Res 2010; 20 (9): 1297-1303.

12. McLaren W, Gil L, Hunt SE et al. The Ensembl Variant Effect Predictor. Genome Biol 2016; 17 (1): 122.

13. Lek M, Karczewski KJ, Minikel EV et al. Analysis of protein-coding genetic variation in 60,706 humans. Nature 2016; 536 (7616): 285-291.

14. Begay RL, Graw S, Sinagra G et al. Role of Titin Missense Variants in Dilated Cardiomyopathy. J Am Heart Assoc 2015; 4 (11).

15. Walsh R, Thomson KL, Ware JS et al. Reassessment of Mendelian gene pathogenicity using 7,855 cardiomyopathy cases and 60,706 reference samples. Genet Med 2017; 19 (2): 192-203.

16. Landrum MJ, Lee JM, Benson $\mathbf{M}$ et al. ClinVar: public archive of interpretations of clinically relevant variants. Nucleic Acids Res 2016; 44 (D1): D862-868.

17. Li Q, Wang K. InterVar: Clinical Interpretation of Genetic Variants by the 2015 ACMG-AMP Guidelines. Am J Hum Genet 2017; 100 (2): 267-280.

18. Francioli LC, Menelaou A, Pulit SL et al. Whole-genome sequence variation, population structure and demographic history of the Dutch population. Nature Genetics 2014; 46 (8): 818-825.

19. Stenson PD, Mort M, Ball EV, Shaw K, Phillips A, Cooper DN. The Human Gene Mutation Database: building a comprehensive mutation repository for clinical and molecular genetics, diagnostic testing and personalized genomic medicine. Hum Genet 2014; 133 (1): 1-9.

20. Watkins H, Rosenzweig A, Hwang DS et al. Characteristics and prognostic implications of myosin missense mutations in familial hypertrophic cardiomyopathy. N Engl J Med 1992; 326 (17): 1108-1114.

21. Rosenzweig A, Watkins H, Hwang DS et al. Preclinical diagnosis of familial hypertrophic cardiomyopathy by genetic analysis of blood lymphocytes. N Engl J Med 1991; 325 (25): 1753-1760.

22. Posen BM, Moolman JC, Corfield VA, Brink PA. Clinical and prognostic evaluation of familial hypertrophic cardiomyopathy in two South African families with different cardiac beta myosin heavy chain gene mutations. Br Heart J 1995; 74 (1): 40-46.

23. Arbustini E, Fasani R, Morbini P et al. Coexistence of mitochondrial DNA and beta myosin heavy chain mutations in hypertrophic cardiomyopathy with late congestive heart failure. Heart 1998; 80 (6): 548-558. 
24. Greber-Platzer S, Marx M, Fleischmann C, Suppan C, Dobner M, Wimmer M. Beta-myosin heavy chain gene mutations and hypertrophic cardiomyopathy in Austrian children. J Mol Cell Cardiol 2001; 33 (1): 141-148.

25. Kassem H, Azer RS, Saber-Ayad M et al. Early results of sarcomeric gene screening from the Egyptian National BA-HCM Program. J Cardiovasc Transl Res 2013; 6 (1): 65-80.

26. Richard P, Charron P, Carrier L et al. Hypertrophic cardiomyopathy: distribution of disease genes, spectrum of mutations, and implications for a molecular diagnosis strategy. Circulation 2003; 107 (17): 2227-2232.

27. Woo A, Rakowski H, Liew JC et al. Mutations of the beta myosin heavy chain gene in hypertrophic cardiomyopathy: critical functional sites determine prognosis. Heart 2003; 89 (10): 1179-1185.

28. Roopnarine O, Leinwand LA. Functional analysis of myosin mutations that cause familial hypertrophic cardiomyopathy. Biophys J 1998; 75 (6): 3023-3030.

29. Mohiddin SA, Begley DA, McLam E et al. Utility of genetic screening in hypertrophic cardiomyopathy: prevalence and significance of novel and double (homozygous and heterozygous) beta-myosin mutations. Genet Test 2003; 7 (1): 21-27.

30. Van Driest SL, Jaeger MA, Ommen SR et al. Comprehensive analysis of the beta-myosin heavy chain gene in 389 unrelated patients with hypertrophic cardiomyopathy. J Am Coll Cardiol 2004; 44 (3): 602-610.

31. Song $\mathbf{L}$, Zou $\mathbf{Y}$, Wang $\mathbf{J}$ et al. Mutations profile in Chinese patients with hypertrophic cardiomyopathy. Clin Chim Acta 2005; 351 (1-2): 209-216.
32. Ingles J, Doolan A, Chiu C, Seidman J, Seidman C, Semsarian C. Compound and double mutations in patients with hypertrophic cardiomyopathy: implications for genetic testing and counselling. J Med Genet 2005; 42 (10): e59.

33. Zou Y, Wang J, Liu X et al. Multiple gene mutations, not the type of mutation, are the modifier of left ventricle hypertrophy in patients with hypertrophic cardiomyopathy. Mol Biol Rep 2013; 40 (6): 3969-3976.

34. Lopes LR, Zekavati A, Syrris $P$ et al. Genetic complexity in hypertrophic cardiomyopathy revealed by high-throughput sequencing. J Med Genet 2013; 50 (4): 228-239.

35. Gruver EJ, Fatkin D, Dodds GA et al. Familial hypertrophic cardiomyopathy and atrial fibrillation caused by Arg663His beta-cardiac myosin heavy chain mutation. Am J Cardiol 1999; 83 (12A): 13H-8H.

36. Lan F, Lee AS, Liang $P$ et al. Abnormal calcium handling properties underlie familial hypertrophic cardiomyopathy pathology in patient-specific induced pluripotent stem cells. Cell Stem Cell 2013; 12 (1): 101-113.

37. Akinrinade $\mathbf{O}$, Ollila L, Vattulainen S et al. Genetics and genotypephenotype correlations in Finnish patients with dilated cardiomyopathy. Eur Heart J 2015; 36 (34): 2327-2337.

38. Glotov AS, Kazakov SV, Zhukova EAet al. Targeted next-generation sequencing (NGS) of nine candidate genes with custom AmpliSeq in patients and a cardiomyopathy risk group. Clin Chim Acta 2015; 446: 132-140.

39. Jaafar N, Girolami F, Zairi I, Kraiem S, Hammami M, Olivotto I. Genetic profile of hypertrophic cardiomyopathy in Tunisia: Is it different? Glob Cardiol Sci Pract 2015; 2015: 16. 